\title{
Influência da Diabetes mellitus tipo II na Osseointegração
}

\author{
Influence of Diabetes mellitus type II on Osseointegration \\ Influencia de la Diabetes mellitus tipo II en la Osteointegración
}

Recebido: 29/07/2021 | Revisado: 02/08/2021 | Aceito: 04/08/2021 | Publicado: 09/08/2021

\author{
Lourrane Freitas \\ ORCID: https://orcid.org/0000-0002-2548-7447 \\ Faculdade de Patos de Minas, Brasil \\ E-mail: freitass.lourrane@hotmail.com \\ Henrique Cury Viana \\ ORCID: https://orcid.org/0000-0001-6168-0917 \\ Faculdade de Patos de Minas, Brasil \\ E-mail: curyhenrique@hotmail.com
}

\begin{abstract}
Resumo
A evolução humana permitiu que estratégias para o edentulismo parcial e total fossem desenvolvidas e aperfeiçoadas. Nesse sentido, a implantodontia contemporânea tem permitido que a reabilitação oral esteja acessível à sociedade, contribuindo para uma melhor qualidade de vida no âmbito estético, alimentar e social. Para tanto, as condições gerais do paciente são de suma importância para o sucesso dos processos para os implantes dentários. Assim, objetivou-se nessa revisão relacionar diversas fontes em busca de informações sobre as dificuldades encontradas na cicatrização óssea em pacientes portadores de diabete mellitus II que se submetem à implantes ósseos. Realizou-se buscas em livros, artigos científicos, revistas eletrônicas e estudos de mestrado e doutorado. Todos os trabalhos utilizados como base de pesquisa relatam como resultado diversos fatores que influenciam à não manutenção da homeostase na fisiologia da cicatrização pela diabetes mellitus tipo II, podendo muitos implantes não obter o sucesso desejado. Os trabalhos avaliados relatam boas experiências no processo de osseointegração apesar das dificuldades orgânicas promovidas pela diabetes mellitus tipo II. Contudo, fica evidente a necessidade de controle desta endocrinopatia para que os procedimentos alcancem a cicatrização almejada.
\end{abstract}

Palavras-chave: Cicatrização; Diabetes mellitus; Implantação dentária; Osseointegração.

\begin{abstract}
Human evolution has allowed strategies for partial and total edentulism to be developed and perfected. In this sense, contemporary implantology has allowed oral rehabilitation to be accessible to society, contributing to a better quality of life in the aesthetic, food and social spheres. Therefore, the general conditions of the patient are of paramount importance for the success of the processes for dental implants. Thus, the aim of this review was to relate several sources in search of information about the difficulties encountered in bone healing in patients with diabetes mellitus II who undergo bone implants. Searches were carried out on books, scientific articles and electronic journals. All works used as a research base report as a result several factors that influence the non-maintenance of homeostasis in the healing physiology of type II diabetes mellitus, and many implants may not achieve the desired success. The evaluated works report good experiences in the osseointegration process despite the organic difficulties caused by type II diabetes mellitus. However, it is evident the need to control this endocrinopathy so that the procedures achieve the desired healing.
\end{abstract}

Keywords: Wound healing; Diabetes mellitus; Dental implantation; Osseointegration.

\section{Resumen}

La evolución humana permitió desarrollar y perfeccionar estrategias para el edentulismo parcial y total. En este sentido, la implantología contemporánea ha permitido que la rehabilitación oral sea accesible a la sociedad, contribuyendo a una mejor calidad de vida en los ámbitos estético, alimentario y social. Por tanto, las condiciones generales del paciente son de suma importancia para el éxito de los procesos de implantes dentales. Así, el objetivo de esta revisión fue enumerar varias fuentes en busca de información sobre las dificultades encontradas en la cicatrización ósea en pacientes con diabetes mellitus II que se someten a implantes óseos. Se realizaron búsquedas en libros, artículos científicos, revistas electrónicas y estudios de maestría y doctorado. Todos los artículos utilizados como base para la investigación informan como resultado varios factores que influyen en el no mantenimiento de la homeostasis en la fisiología de la curación debido a la diabetes mellitus tipo II, y muchos implantes pueden no lograr el éxito deseado. Los trabajos evaluados relatan buenas experiencias en el proceso de osteointegración a pesar de las dificultades orgánicas provocadas por la diabetes mellitus tipo II. Sin embargo, es evidente la necesidad de controlar esta endocrinopatía para que los procedimientos logren la curación deseada.

Palabras clave: Curación; Diabetes mellitus; Implantación dental; Osteointegración. 


\section{Introdução}

Segundo Sakakura, Margonar e Marcantonio Júnior (2005), desde as civilizações mais antigas o homem procura por estratégias de repor a dentição perdida, ou seja, solucionar o edentulismo parcial ou total. Fernandes Junior, et al., (2014), destacam-se que nesse sentido, ao longo do tempo, a odontologia foi se desenvolvendo tendo como um dos marcos o surgimento da implantodontia. Silva e Filho (2017) cita que essa especialidade é uma opção para a reabilitação oral, promovendo uma terapêutica por meio da restauração da estabilidade oclusal com a realização de implantes dentários osseointegráveis.

Paraguassu et al., (2019), a partir da descoberta da osseointegração em 1952 pelo professor Branemark, essa técnica foi definida como uma conexão direta, estrutural e funcional entre o osso vivo e a superfície de um implante submetido à carg a funcional. (Bränemark et al., 1987). Contudo, a formação da interface osso-implante é complexo e é envolvida por diversos fatores: material, forma, topografia, química de superfície, além da carga mecânica e técnica cirúrgica, podendo encontrar variáveis individuais, como quantidade e qualidade óssea que afetam a osseointegração, (Schimiti \&Junior, 2010).

De acordo com Klokkevold e Mealey, (2007), a Diabetes Mellitus é considerada um transtorno metabólico complexo, tendo como característica fisiopatológica a hiperglicemia crônica. Contudo, a produção reduzida ou a completa inibição de insulina pode corroborar para que o transporte de glicose para dentro das células não aconteça da forma esperada, isto é, gerando um acúmulo de glicose no sangue e na urina. Além dessa definição geral, esta endocrinopatia é classificada como tipo 1 (resultante da destruição da insulina produzida através das células $\beta$ do pâncreas pelo próprio organismo) e tipo 2 (proveniente da redução da ação da insulina ou pelo aumento na concentração de glicose no sangue). (Mealey \& Moritz, 2003).

Diante desse cenário, a população tem se interessado pelos recursos reabilitadores, evidenciando a importância da avaliação geral de todos os indivíduos. (Balshi \&Wolfinger, 1999). Para isso, é necessário que o dentista tenha um diagnóstico criterioso, elaborado a partir de exames clínicos, exames laboratoriais complementares, identificação de fatores de risco e estabelecimento de um plano de tratamento. (Freitas et al., 2020). O Ministério da Saúde do Brasil. (2013), cita que entre estes fatores, a diabetes mellitus tipo 2 (DM-II) tem se destacado entre os pacientes brasileiros, atingindo 12 milhões de pessoas, segundo a Pesquisa Nacional de Saúde realizada pelo Ministério da Saúde.

A técnica cirúrgica de implante dentário é um procedimento largamente utilizado na clínica de reabilitação oral, sendo classificado como seguro e previsível. Entretanto, fatores individuais de risco locais e sistêmicos podem influenciar em maiores taxas de falha. (Silva et al., 2020). Diante desse cenário, Marconi e Lakatos (2007), concluiu que tem-se registrado em literatura científica dificuldades para o processo cicatricial em especial óssea em pacientes portadores de diabete mellitus II, visto que esta patologia é comum na clínica médica no Brasil, assim, entender as influências dessa endocrinopatia no sucesso terapêutico é necessário para a evolução da terapêutica odontológica.

Essa revisão teve como objetivo relacionar diversas fontes em busca de informações sobre as dificuldades encontradas na cicatrização óssea em pacientes portadores de diabetes mellitus II, além de estabelecer os critérios de cicatrização frente a instalação de implantes e definir fisiologicamente o processo de osseointegração na instalação de implantes em pacientes portadores desta endocrinopatia.

\section{Metodologia}

Este estudo será fundamentado na abordagem qualitativa de acordo com e Marconi e Lakatos (2007), a pesquisa qualitativa é caracterizada por sua investigação volvida para os fatos qualitativos. As fontes utilizadas serão livros, artigos científicos, revistas eletrônicas, estudos de mestrado e doutorado. O critério de seleção das fontes de pesquisa versará publicações dos últimos 10 anos, com exceção dos livros, para os quais não se estabelecerá restrições desta natureza.

Na primeira análise utilizando as palavras-chaves "diabetes mellitus" e "osseointegração" encontramos 252 trabalhos. 
Aplicando os filtros de pesquisa temporal e espacial o número foi reduzido para 117. Definindo os critérios de inclusão (indivíduos diabéticos na clínica odontológica) e exclusão (indivíduos hígidos na clínica odontológica) chegou-se a 64 trabalhos, os quais serão analisados nesta revisão sistemática.

Para tanto, será realizado a leitura dos artigos que possuam concreta relação com o tema deste trabalho retirando informações que vão compor esta revisão. Serão utilizados para a busca os repositórios on-line Scielo, PubMed, usando como palavras-chave "osseointegração", "diabetes mellitus II", "implantes dentários".

\section{Revisão de Literatura}

Assunção et al., (2001), refere-se que o DM se qualifica como um problema pessoal e de saúde pública com proporções importantes quanto à magnitude e à transcendência, apesar dos progressos no campo da investigação e atenção aos pacientes. Assim, a diabetes mellitus é a patologia endócrina com mais causas de morbidade e mortalidade entre os brasileiros.

Conforme Lima et al., (2012), esta é uma síndrome de caráter crônico metabólica causada pela alteração da secreção de insulina. Sintetizada no pâncreas, a insulina é o hormônio responsável pela redução da glicemia, ao promover a entrada de glicose nas células. (American Diabetes Association. ADA, 2019)

De acordo com o estudo de Sonis et al., (1996), a diabetes afeta 17 em cada 1000 pessoas entre 25 e 44 anos, e 79 indivíduos em 1000 com idade superior aos 75 anos. A Federação Nacional de Diabetes (FID) registrou em 2017, 425 milhões de portadores da doença por todo o mundo, o que desperta aos profissionais de saúde preocupação quanto aos desdobramentos da doença na humanidade. (ATLAS, IDF Diabetes, 2017). Além disso, a mesma instituição prevê um aumento de $48 \%$ no número de casos em 2045.

A diabetes mellitus (DM) é classificada de acordo com a sua etiologia e não com o tipo de tratamento indicado. Assim, tem-se quatro tipos de classes clínicas de diabetes conforme Quadro 1:

Quadro 1 - Classes clínicas e características gerais da diabetes pela Associação Americana de Diabetes.

\begin{tabular}{|c|l|}
\hline Classes clínicas da diabetes & Características gerais \\
\hline DM tipo 1 & $\begin{array}{l}\text { Ocorre uma destruição das células } \beta \text { no pâncreas, geralmente levando a uma deficiência total } \\
\text { de insulina. }\end{array}$ \\
\hline DM tipo 2 & $\begin{array}{l}\text { Resulta de um defeito secretor de insulina, ou as células receptoras de insulina não } \\
\text { reconhecem esse hormônio. }\end{array}$ \\
\hline DM gestacional & Possui ocorrência no período de gravidez. \\
\hline Outros tipos de DM & $\begin{array}{l}\text { Ocorre devido a outras causas como: defeitos genéticos na função das células } \beta, \text { defeitos } \\
\text { genéticos na ação da insulina, doenças do pâncreas exócrino (fibrose cística) e o tipo induzido } \\
\text { por drogas ou químicos (por exemplo, no tratamento de HIV/AIDS ou após transplante de } \\
\text { órgãos). }\end{array}$ \\
\hline
\end{tabular}

Fonte: ADA. American Diabetes Association. (2019).

O estado de cronicidade da hiperglicemia causa modificações no sistema imunológico do paciente, aumentando o nível de citocinas pró-inflamatórias, resultando em uma maior susceptibilidade do surgimento de novas doenças. (Gomes \& Accardo, 2019). Assim, as consequências quando a doença está instalada é o aumento da concentração da glicose no sangue e a alteração no metabolismo dos lipídios. (Harrison et al., 2002)

Goodson e Hunt (1979), Cianciola et al., (1982) e Pilatti et al., (1997), reference que algumas complicações sistêmicas podem ser registradas quando a doença não possui um controle efetivo pelo paciente, como a cegueira (retinopatia), doença renal (nefropatia), problemas cardíacos devido à acidentes vasculares, maior prevalência da doença periodontal. Além dessas, 
a diabetes mellitus causa baixa perfusão tecidual e os seus portadores apresentam problemas relacionados à cicatrização. (Gross et al., (2002) \& Cavalcanti S, 2008).

De acordo com Lima e Araújo (2013), quando se tem o surgimento de uma ferida em qualquer local do corpo, o organismo desencadeia uma cascata de reações celulares e bioquímicas com o propósito de reparar o tecido injuriado. Contudo, Olson et al (2000), mencionam que em pacientes diabéticos, este reparo é lentificado. Um processo que possui sua eficiência bastante comprometida por esta doença são os implantes ósseointegrados. A previsibilidade desses procedimentos em pacientes hígidos tem taxas de sucesso de $78 \%$ a $97 \%$. No entanto, a osseointegração permanece duvidosa e demanda cuidado intensivos do profissional para os pacientes com problemas sistêmicos, como, o diabetes.

As principais alterações bucais dos diabéticos são: hipoplasia, hipocalcificação do esmalte, diminuição do fluxo e aumento da acidez e da viscosidade salivar, que são fatores de risco para a formação de cárie. (Dinato \& Polido, 2001). Além disso, Sousa et al., (2003), citam que o grande conteúdo de glicose e cálcio na saliva estimulam o aumento na quantidade de cálculo dentário e fatores irritantes nos tecidos. Tem-se relatos da ocorrência também de xerostomia, glossodínia (ardor na língua), eritema e distúrbios da gustação. (Alves et al., 2006).

Nesse sentido, Oates et al., (2009), concluem que a DM sendo uma contraindicação ao uso de terapias com implantes devido à cicatrização dificultosa e à resposta imune comprometida, estando o controle glicêmico diretamente relacionado à estabilidade do implante. Os resultados são satisfatórios quando esta condição sistêmica está bem controlada, principalmente quando associados com tratamentos de superfície dos implantes. (Schelegel et al., 2013).

Segundo, Takeshita et al., (1997), fisiologicamente, as estruturas pelos quais o doente com Diabetes Mellitus descompensado pode diminuir o índice de osso ao redor dos implantes é a redução de íons cálcio no sangue, aumento de cálcio e fosfato na urina e a produção reduzida do colágeno. Além disso, El Askary, et al., (1999), citam que a hiperglicemia aumenta a secreção de um hormônio da paratireoide, o qual estimula os osteoclastos. Ainda nessa perspectiva, a secreção do hormônio do crescimento é diminuta quando a concentração da insulina sérica é baixa. (Nevins et al., 1998).

Conforme Damasceno et al., (2012), a DM é uma doença complexa, que demanda mudanças no estilo de vida e no comportamento diário das pessoas por ela acometidas e necessita de uma comunicação efetiva entre paciente e profissional.

\section{Discussão}

O presente estudo foi construído a partir de referências científicas que mostraram que o insucesso do implante dentário está relacionado com o diabetes descompensado. Todavia, a literatura revisada apresentou resultados divergentes quanto à influência da DM - 2 e a efetividade dos implantes osseointegrados, evidenciando que os estudos não são unânimes.

Almeida et al., (2017) e Valle et al., (2017), afirmam que a fisiopatologia do Diabetes Mellitus na homeostase oral, essa patologia versa todo o processo de cicatrização, formação e remodelamento ósseo, tornando-se uma condição a ser considerada na implantodontia. Diante desse cenário, existe a necessidade do controle glicêmico para o sucesso do tratamento.

King et al., (2016), dizem que a hiperglicemia afeta a renovação óssea e a qualidade da matriz orgânica de forma negativa, resultando numa deterioração da qualidade, resiliência e estrutura do tecido ósseo. Seguindo essa perspectiva, Lee et al., (2016), citam que o controle glicêmico é ineficaz, ou seja, pacientes que possuem a patologia em descompensação pode ser considerada uma contraindicação para a terapia com implantes dentários, visto o maior risco de cicatrização tardia, infecções e complicações vasculares.

Ainda nessa temática, não há nenhum tipo de fator de risco no tratamento com implante para pacientes diabéticos com bom controle glicêmico, não havendo em seu estudo nenhuma diferença significativa nas taxas de sucesso e sobrevida dos implantes. No entanto, neste estudo, não foram encontradas correlações entre os níveis plasmáticos de glicose por meio da hemoglobina glicada (HbA1c) e sucesso ou sobrevida dos implantes. (Cabrebra-Domínguez et al., 2017). 
A realização de técnicas de implantes dentários é recomendada aos pacientes que possuem um controle glicêmico dentro ou próximo da normalidade, além de possuir a área em condições favoráveis para o procedimento, isto é, apresentando boa quantidade e qualidade óssea, corroborando com os autores supracitados. (Naujokat H et al., (2016) \& Santos JM. (2018). Entretanto, há a contraindicação da diabetes mellitus para a implantoterapia, estando relacionada ao controle glicêmico, mostrando que a colocação de implantes dentários em pacientes com diabetes com controle adequado é tão bem sucedida como na população em geral. (Beikler \& Flemmig, 2003).

Diante da condição de portador da DM, a colocação de implantes em doentes tem sido um tema discutido na odontologia, pois a prevalência da diabetes tem aumentado a cada ano, desta forma, há mais diabéticos que procuram o implante como forma de reabilitação. (Esteves, 2019).

Além disso, devolver função, estética, autoestima, beleza, fonética e saúde aos pacientes são propósitos da implantodontia. (Freira et al., 2018).

Assim, todos esses dados corroboram para a necessidade de novas pesquisas e estudos clínicos que visem a modernização nos protocolos para esses pacientes. Para tanto, estudos em animais têm sido amplamente aplicados visando o melhor entendimento de todo o processo fisiológico da cicatrização de implantes mediante à DM.

Nesse aspecto, o início da proliferação celular e a diferenciação dos osteoblastos no osso diabético de animais foram prolongados quando comparados com o osso de um animal em caráter de normalidade glicêmica. (Colombo et al., 2011). Outro autor corroborou que o diabetes provocou alterações histopatológicas e estruturais na osseointegração de implantes no fêmur de ratos diabéticos, tendo uma melhora de resultados quando ocorria a instituição de insulinoterapia. Ambos os estudos citados comprovam atraso na cicatrização e na estrutura do osso diabético, o que pode ser comparado com o humano. (Souza, 2011).

\section{Conclusão}

O diabetes não é uma contraindicação absoluta para a colocação de implantes dentários. O importante é o paciente a ser implantado nestas condições mantê-la controlada especialmente durante o período de osseointegração e que haja a administração de antibióticos antes e após a cirurgia e ainda a prescrição de bochechos de clorexidina a $0,12 \%$ durante duas semanas após o procedimento cirúrgico para evitar intercorrências no período de cicatrização.

\section{Referências}

ADA. American Diabetes Association. (2019). Classification and Diagnosis of Diabetes: Standards of Medical Care in Diabetes-2019. Diabetes Care, 42(1), 13-28 https://www.archhealthinvestigation.com.br/ArcHI/article/view/279.

Adell, R., Lekholm, U., Rockler, B., \& Branemark, P. I. (1981). A 15-years study ofosseointegrated implants in the treatment of the endentulous jaw. International Journal Oral Surgery, 10(6), 387-416. https://pubmed.ncbi.nlm.nih.gov/6809663/.

Almeida, J. F., Pauferro, B. C. S., Saraiva, A. I., \& Oliveira Junior, V. F. (2017). Implantes dentários e a osseointegração em paciente diabéticos, 11 FEPEG, Universidade, Sociedade e Políticas Públicas. http://www.fepeg2017.unimontes.br/anais/download/321.

Alves, C., Brandão, M., Andion, J., Menezes, R., \& Carvalho, F. (2006). Atendimento odontológico do paciente com diabetes mellito: recomendações para a prática clínica. Revista Científica Medicina Biológica, 5:2, 97-110. https://periodicos.ufba.br/index.php/cmbio/article/view/4116.

Assunção, M. C. F., Santos, I. S. \& Gigantea, D. P. (2001). Atenção Primária em Diabetes no Sul do Brasil: estrutura, processo e resultado. Revista de Saúde Pública, 35(1), 88-95.

Atlas, IDF Diabetes. (2017). Brussels, Belgium: International Diabetes Federation (IDF), 8ª ed., p. 147, 2017.

Azevedo, A. P., Papelbaum, M., \& D'elia, F. (2002). Diabetes and eating disorders: a high Diabetes and eating disorders: a high-risk Association.Revista Brasileira de Psiquiatria, 24, 77-80. https://www.scielo.br/j/rbp/a/x8QpnnWcjC59Vhq3rVJKPYB/abstract/?lang=en.

Balshi, T. J., \& Wolfinger, G. J. (1999). Dental implants in the diabetic patient: a retrospective study. Implant Dent, 8, 355-9. https://pubmed.ncbi.nlm.nih.gov/10709480/. 
Bränemark, P. I., Zarb, G. A., \& Albrektsson, T. (1987). Protesis tejido-integradas: la osseointegration en la odontologia clínica, 350.

Branemark, P. I., et al (1969). Intra-osseous anchorage of dental prostheses. I. Experimental studies. Scand Journal Plastic Reconstruction Surgery, 2(3), 81100. https://pubmed.ncbi.nlm.nih.gov/4924041/.

Beikler, T., \& Flemmig, T. F. (2003). Implants in the medically compromised patient. CritRev Oral BiolMed, 14 , 305-16. https://pubmed.ncbi.nlm.nih.gov/12907698/.

Brasil. Ministério da Saúde. (2013). Secretaria de Atenção à Saúde. Departamento de Atenção Básica. Estratégias para o cuidado da pessoa com doença crônica: diabetes mellitus.

Cabrebra-Domínguez, J. J., et al (2017). A Prospective Case-Control Clinical Study of Titanium-Zirconium Alloy Implants with a Hydrophilic Surface in Patients with Type 2 Diabetes Mellitus. International Journal of Oral \& Maxillofacial Implants, 32 (5). https://pubmed.ncbi.nlm.nih.gov/28906508/.

Cardoso, A. L., et al (2006). Dental implants in Diabetic Patients, Innovations Implant Journal, 1 (2), 47-52. https://bjihs.emnuvens.com.br/bjihs/article/view/14.

Cianciola, L. J., et al (1982). Prevalence of periodontal disease in insulindependent diabetes mellitus (juvenile diabetes). Journal Am Dentistry Associated, 104, 653-60. https://pubmed.ncbi.nlm.nih.gov/7042797/.

Campos Junior, A., \& Passanezi, E. (1996). Por que a osseointegração revolucionou a Implantodontia? In: Todescan FF, Botino MAC. (1996). Atualização na clínica odontológica: a prática da clínica geral. Artes Médicas, 249-97.

Cavalcanti, S. (2008). Complicações crônicas do diabetes. Sociedade Brasileira de Diabetes. http://www.diabetes.org.br/tudo-sobre--diabetes/complicacoescronicas.

Colombo, J. S., et al. (2011). Delayed osteoblast differentiation and altered inflammatory response around implants placed in incisor sockets of type 2 diabetic rats. Clin Oral Impl. Res, 22, 578-586.

Courtney, M. W., Snider, T. N., \& Cottrell, D. A. (2010). Dental Implant Placement in Yype II Diabetics: A Review of Literature, Journal of the Massachusetts Dental Society, 59 (1), 12-14. https://pubmed.ncbi.nlm.nih.gov/20521447/.

Damasceno, M. M. C., et al (2012). A comunicação terapêutica entre profissionais e pacientes na atenção em diabetes mellitus. Revista Latino-Americana em Enfermagem, 20(4). https://www.scielo.br/j/rlae/a/PwLnQG39GskmzRTtdG9F3Sr/?lang=pt\&format=pdf.

Dinato, J. C., \& Polido, W. D. (2001). Implantes osseointegrados cirurgia e prótese. São Paulo: Artes Médicas Divisão Odontológica.

El Askary, A. S., et al (1999). Why do dental implants fail? Part I. Implant Dent, 8, 2, 173-83. https://journals.lww.com/implantdent/abstract/1999/02 000/why_do_dental_implants_fail_part_i.11.aspx.

Esteves, A. F. V. (2019). A influência da diabetes mellitus na osseointegração [TCC] São Paulo: Instituto Universitário Egas Moniz.

Farias, I. O. B., \& Freitas, M. A. (2017). Aplicação do laser no tratamento da periimplantite. Revista Bahiana de Odontologia, 8 (4), 141-151. https://www.researchgate.net/publication/321893342_APLICACAO_DO_LASER_NO_TRATAMENTO_DA_PERIIMPLANTITE.

Faverani, L. P., et al. (2011). Implantes ósseointegrados: evolução e sucesso, 30(1), 47-58. https://repositorio.unesp.br/handle/11449/133333.

Fernandes Junior, R. C., et al (2014). Próteses totais fixas sobre implante com carga imediata em mandíbula. Rev. de Inic. Cient. da Univers. Vale do Rio Verde. Disponível em: https://core.ac.uk/download/pdf/230549989.pdf.

Freira, C. N. B. M., et al. (2018). Complicações decorrentes da reabilitação com implantes dentários. Revista Uningá, 51 (3). http://revista.uninga.br/index.php/uninga/article/view/1361.

Fiorellini, J. P., \& Nevins, M. L. (2000). Dental implant considerations in the diabetic patient, Periodontology, 23, 73-77. https://pubmed.ncbi.nlm.nih.gov/11276767/.

Freitas, E. G., et al. (2020). Influência da diabetes na implantodontia.Revista de Odontologia Contemporânea, 4(1), 27-41. http://www.rocfpm.com/index.php/revista/article/view/449.

Friggi, T. R., et al.. (2011). Laserterapia aplicada à implantodontia: análise comparativa entre diferentes protocolos de irradiação. Innovations Implant Journal: Biomaterial sand Esthetics, 6 (1), 44-48. https://pesquisa.bvsalud.org/portal/resource/pt/lil-618565.

Genovese, W. J. (2007). Laser de baixa intensidade: aplicações terapêuticas. 2. Ed. São Paulo: Santos.

Gomes, B. F., \& Accardo, C. M. (2019). Mediadores imunoinflamatórios na patogênese do diabetes mellitus. Einstein (São Paulo), 17:1, p. 1-5. https://www.scielo.br/j/eins/a/NDX97nHRTyZJzW9ssNpHfWP/?format=pdf\&lang=pt.

Goodson, W. H., \& Hunt, T. K. (1979). Wound healing and the diabetic patient. Surgery Gynecolology Obstetry, 149, 600-7. https://europepmc.org/article/med/2179891.

Gross, J. L., et al. (2002). Diabetes melito: diagnóstico, classificação e avaliação do controle glicêmico. Arquivo Brasileiro Endocrinologia Metabólica, 46:1, 16-26. https://www.scielo.br/j/abem/a/vSbC8y888VmqdqF7cSST44G/abstract/?lang=pt.

Harrison T. R. et al. (2002). Medicina Interna. (15a ed.), Mc Graw-Hill, v. 1.

Jesus, L. K. (2017). Avaliação dos efeitos do laser de baixa intensidade na osseointegração de implantes com diferentes superfícies: análise por frequência de ressonância e biomecânica [TCC]. São Paulo: Faculdade de Odontologia de Araçatuba da Universidade Estadual Paulista "Júlio de Mesquita Filho". 
King, S., et al. (2016). The effect of hyperglycaemia on osseointegration: a review of animal models of diabetes mellitus and titanium implant placement. Archives of Osteoporosis, 11 (1), 29. https://pubmed.ncbi.nlm.nih.gov/27637755/.

Klokkevold, P. R., \& Mealey, B. L. (2007). Influência das Doenças Sistêmicas e do Estresse sobre o Periodonto.In: Newman MG, Takei H. H, Klokkevold P. R., Carranza F. (2007). A Periodontia Clínica.10a ed. Rio de Janeiro: Elsevier, 284-312. https://bjihs.emnuvens.com.br/bjihs/article/view/14.

Mealey, B. L., \& Moritz, A. J. (2003). Hormonal influences: effects of diabetes mellitus and endogenous female sex steroid hormones on the periodontium. Periodontol, 32(1), 59-81. https://pubmed.ncbi.nlm.nih.gov/12756034/.

Kuzyk, P. R. T., et al. (2010). A biodegradable scaffold for the treatment of a diaphyseal bone defect of the tibia. Journal Orthop Res, 28 (4), 474-480. https://pubmed.ncbi.nlm.nih.gov/19859898/.

Lee, J. S., et al. (2016). Effects of glycemic control in patients with type II diabetes mellitus on dental implant survival. Journal of Oral and Maxillo facial Surgery, 74 (9). https://www.joms.org/article/S0278-2391(16)30379-2/abstract.

Lima, M. H. M., \& Araújo, E. P. (2013). Diabetes mellitus e o processo de cicatrização cutânea. Cogitare Enfermagem, 18, 1, 170-72. https://revistas.ufpr.br/cogitare/article/view/31323/20032.

Lima, M. H., et al. (2012). Topical insulin accelerates wound healing in diabetes by enhancing the AKT and ERK pathways: A double-blind placebocontrolled clinical trial. PloS, 7:5. http://www.plosone.org/article/authors/info\%3Adoi\%2F10.1371\%2Fjournal.pone.0036974.

Luís, A. A. (2013). Efeitos do laser de baixa potência no processo de cicatrização de feridas cutâneas: revisão de literatura [TCC]. Formiga: Centro Universitário de Formiga.

Marconi, M. A., \& Lakatos, E. M. (2007). Fundamentos de metodologia científica. (6a ed.), Atlas.

Martins V, et al. (2011). Osseointegração: análise de fatores clínicos de sucesso e insucesso. Revista Odontológica de Araçatuba, 32 (1), 26-31. https://apcdaracatuba.com.br/revista/v32n12011/TRABALHO4.pdf.

Mendes, V. C., \& Davies, J. E. (2016). Uma nova perspectiva sobre a biologia da osseointegração. Revista Associada Paulista de Cirurgiões Dentistas, 70(2), 166-171. http://revodonto.bvsalud.org/scielo.php?pid=S0004-52762016000200011\&script=sci_arttext\&tlng=pt.

McNally, A. K., \& Anderson, J. M. (2011). Macrophage fusion and multinucleated giant cells of inflammation. Adv Exp Med Biol, 713, 97-111. https://pubmed.ncbi.nlm.nih.gov/21432016/.

Miranda, T. A. C., et al. (2018). A influência do fumo na reabilitação com implantes osseointegrados: revisão de literatura. Revista de Odontologia da Universidade Cidade de São Paulo, 30 (2), 169-176. https://publicacoes.unicid.edu.br/index.php/revistadaodontologia/article/view/680.

Misch, C. E. (2000). Implantes dentários contemporâneos. (2a ed.), Ed. Santos, 21-32.

Morris, H. F., et al. (2000). Implant survival in patients with type 2 diabetes: placement to 36 months, Ann, 5, 157-165. https://pubmed.ncbi.nlm.nih.gov/11885176/.

Naujokat, H., Kunzendorf, B., \& Wiltfang, J. (2016). Dental implants and diabetes mellitus - a systematic review. International Journal of Implant Dentistry, 2 (1). https://www.ncbi.nlm.nih.gov/pmc/articles/PMC5005734/.

Nevins, M. L., et al. (1998). Wound Healing Around Endosseous Implants in Experimental Diabetes. International Journal Oral Maxillofac Implant, 13:5, 620629. https://pubmed.ncbi.nlm.nih.gov/9796145/.

Oates, T, et al. (2009). Glycemic Control and Implant Stabilization in Type 2 Diabetes Mellitus. Journal of Dental Research, 88:4, 367-71. https://pubmed.ncbi.nlm.nih.gov/19407159/.

Olson, J. W., et al. (2000). Dental endosseous implant assessments in a type 2 diabetic population: a prospective study. International Journal Oral Maxillofac Implants, 15, 811-8, 2000.

Paraguassu, E. C., et al. (2019) Quality of life and satisfaction of users of total tissue-supported and implant-supported prostheses in the municipality of Macapá, Brazil.Journal of Development Research. https://www.journalijdr.com/quality-life-and-satisfaction-users-total-tissue-supported-and-implantsupported-prostheses.

Pilatti, G. L., et al. (1997). Avaliação dos parâmetros clínicos da doença periodontal em pacientes diabéticos não insulinodependentes submetidos a tratamento periodontal não cirúrgico. Revista Paulista de Odontologia, 19, 8-12. https://pesquisa.bvsalud.org/portal/resource/pt/lil-387233.

Renouard, F \& Rangert, B. (2008). Fatores de risco em implantodontia: Análise clínica simplificada para um tratamento previsível. (2a ed.), Quintessence.

Sakakura, C. E., Margonar, R., \& Marcantonio Júnior, E. (2005). A Influência do Diabetes Mellitus na Implantodontia: uma revisão de literatura. Rev. Inter. Period. Clínica. https://www.dtscience.com/wp-content/uploads/2015/10/A-Influ\%C3\%AAncia-do-Diabetes-Mellitus-na-Implantodontia.-UmaRevis\%C3\%A3o-de-Literatura.pdf.

Santos, J. M. (2018). A influência da diabetes mellitus no processo de osseointegração na implantodontia. Revista de Iniciação Científica da Universidade Vale do Rio Verde.

Schimiti, E., \& Junior A. J. Z. (2010). Análise de osseointegração primária do sistema de implantes.Implant News 7(3), 45-9. http://www.bionnovation.com.ec/pdf/001POR.pdf.

Silva ADF \& Filho JLF. (2017). A influência da Diabetes Mellitus tipo 2 no processo de 34 osseointegração de implantes dentários - Revisão de literatura. Soc. Bras. de Diabetes. https://bdigital.ufp.pt/bitstream/10284/2592/3/TM_16594.pdf. 
Research, Society and Development, v. 10, n. 10, e236101018866, 2021

(CC BY 4.0) | ISSN 2525-3409 | DOI: http://dx.doi.org/10.33448/rsd-v10i10.18866

Silva, A. D. F., et al. (2020). Uma revisão literária sobre a influência da diabetes mellitus tipo 2 no processo de osseointegração de implantes dentários.BrazilianJournalof Health Review, 3(4), 11277-11292. https://www.brazilianjournals.com/index.php/BJHR/article/view/15858.

Sonis, S. T., et al. (1996). Princípios e prática de medicina oral. Guanabara Koogan.

Sousa, R. R., et al. (2003). O paciente ondontológico portador de Diabetes Melitus: uma revisão da literatura, Pesquisa Brasileira de Odontopediatria Clínica Integrada, 3:2, 71-77. https://biblat.unam.mx/pt/revista/pesquisa-brasileira-em-odontopediatria-e-clinica-integrada/articulo/o-paciente-odontologico-portadorde-diabetes-mellitus-uma-revisao-da-literatura.

Souza, A. C. R. (2011). Avaliação da formação óssea em implantes dentários de ratos não diabéticos, diabéticos não controlados e controlados com insulina [TCC] São Paulo: Universidade Estadual Paulista, Faculdade de Medicina de Botucatu.

Traini, T., et al.. (2008). Direct laser metal sintering as a new approach to fabrication of an isoelastic functionally graded material for manufacture of porous titanium dental implants. Dent Mater, 24, 1525-1533. https://pubmed.ncbi.nlm.nih.gov/18502498/.

Trindade, R., et al. (2015). Current concepts for the biological basis of dental implants: foreign body equilibrium and osseointegration dynamics. Oral Maxillofac Surg Clin North Am, 27 (2), 175-83. https://pubmed.ncbi.nlm.nih.gov/25753575/.

Takeshita, F., et al. (1997). The effects of diabetes on the interface between hydroxyapatite implants and bone in rat tibia. Journal of Periodontology, 68, 2, 180-185. https://pubmed.ncbi.nlm.nih.gov/9058337/.

Valle, L. S. E. M. B., et al. (2017). DRev o21- Influência do Diabetes Mellitus sobre os tecidos periimplantares. Archives of Health Investigation, 6.

Valero, A. M., et al. (2006). Effects of diabetes on the osseointegration of dental implants, Med Oral Patol Oral Cir Bucal, 12:38-43. https://pubmed.ncbi.nlm.nih.gov/17195826/.

Xavier, K. C. R., et al. (2008). Os efeitos do laser de baixa potência: um levantamento bibliográfico. In: Congresso de Engenharia Biomédica, n. 21, São José dos Campos. XXI Congresso de Engenharia Biomédica, São José dos Campos: Universidade do Vale do Paraíba. 